\title{
Lattice softening in nanometer-size iron particles
}

\author{
J. R. Childress and C. L. Chien \\ Department of Physics and Astronomy, The Johns Hopkins University, Baltimore, Maryland 21218 \\ M. Y. Zhou and Ping Sheng \\ Exxon Research and Engineering Co., Annandale, New Jersey 08801
}

(Received 15 May 1991)

\begin{abstract}
The thermal properties of nanometer-size bcc iron particles imbedded in amorphous $\mathrm{Al}_{2} \mathrm{O}_{3}$ and $\mathrm{SiO}_{2}$ matrices have been investigated by Mössbauer spectroscopy. Contributions from both core and surface Fe atoms are observed in the Mössbauer spectrum. The Debye temperature in the core is seen to decrease dramatically: by more than $30 \%$ for particles smaller than $2 \mathrm{~nm}$. This is good agreement with a recent self-consistent Einstein-model calculation on the dynamics of small Fe particles. Differences in results regarding the lattice spacing, however, suggest a possible role of the matrix in limiting lattice relaxation.
\end{abstract}

\section{INTRODUCTION}

An important area in the study of crystalline matter is the transition region between atomic clusters (containing a few tens of atoms) and bulk solids. In this intermediate region, where crystal sizes are in the nanometer range, the solid-state properties are dominated by surface, interface, and finite-size effects. The crystalline lattice spacing in small particles, for example, can be altered by surface relaxation, stress, or strain. Similarly, the restricted geometry of such systems can exclude long-wavelength phonons and emphasize surface modes, leading to unusual phonon spectra. These finite-size effects play significant roles in determining the lattice dynamics, melting behavior, and superconducting properties of small particles. Despite strong efforts to understand and document these and other effects, particularly for metallic particles, ${ }^{1}$ the study of nanometer-size solids remains an area where there is much to be explored.

In the case of the lattice dynamics, it has been shown ${ }^{2}$ that an elastic-body model of small particles with surface relaxation leads to a "softening" of the phonon spectrum (a shift of the spectrum towards longer wavelengths). In general, however, this kind of continuum model is expected to break down as the particle size becomes very small and quantum size effects can no longer be ignored. In that limit, successful models must take into account the discrete nature of the particle structure.

Experimentally, the effects of finite size on the lattice dynamics are difficult to observe. First of all, the fabrication of nanometer-size particles, their protection from reaction with the atmosphere, and their dispersion into a suitable matrix are challenging requirements. Additionally, to accurately measure the signal from the particles themselves, one must be able to extract the contribution of interest from the often very large background due to the matrix and other irrelevant phases in the specimen. This is the main problem with using methods such as heat-capacity measurements. ${ }^{3}$ The alternative is to use methods that measure the atomic mean-square displacement through a Debye-Waller factor, such as x-ray diffraction ${ }^{4,5}$ or Mössbauer spectroscopy. ${ }^{6}$ In this respect, Mössbauer spectroscopy is an ideal experimental tool, since it only probes the local environment of the absorbing nucleus, allowing a simple and direct comparison between atoms in bulk material and in small particles. Changes in the Debye temperature of gold, tin, and tungsten particles have been previously reported, ${ }^{7,8}$ although the data is sparse and in some cases contradictory. In the case of $\mathrm{Fe}$, Hayashi et al., ${ }^{9}$ have used Mössbauer spectroscopy to study the Debye temperature of 6.6-nm particles bound in a matrix and found no difference from that of bulk Fe. Thus much smaller particle sizes appeared to be a necessary requirement for the possible observation of lattice softening effects in $\mathrm{Fe}$. With this in mind, we have examined by Mössbauer spectroscopy the thermal properties of ultrafine bodycentered-cubic (bcc) $\mathrm{Fe}$ particles in $\mathrm{Fe}-\mathrm{SiO}_{2}$ and $\mathrm{Fe}-\mathrm{Al}_{2} \mathrm{O}_{3}$ granular materials. In this paper, we present experimental evidence for a dramatic decrease in the Debye temperature $\left(\Theta_{D}\right)$ for particles with sizes below $3 \mathrm{~nm}$. This effect is in good agreement with a recently published self-consistent Einstein-model calculation of the meansquare displacement of $\mathrm{Fe}$ atoms in nanometer-size bcc particles, ${ }^{10}$ which we also briefly review.

\section{THEORY}

Since low-frequency phonons with half wavelengths larger than the particle size are absent from free-standing small particles, the Einstein model (where high-frequency modes dominate) is used to evaluate the dynamical behavior of nanometer-size particles. The atoms within a particle are assumed to interact via the Morse potential: ${ }^{11}$

$v\left(x_{i j}\right)=D\left\{\exp \left[-2 \alpha\left(x_{i j}-r_{0}\right)\right]-2 \exp \left[-\alpha\left(x_{i j}-r_{0}\right)\right]\right\}$. 
$D$ and $\alpha$ are material-specific parameters (obtained experimentally from bulk measurements) that determine the strength and range of the interaction, $x_{i j}$ is the separation between atoms $i$ and $j$, and $r_{0}$ is the interatomic distance that minimizes $v\left(x_{i j}\right)$ for a two-atom system. The anharmonicity in $v\left(x_{i j}\right)$ will result, at finite temperatures, in an effective interatomic potential $\Psi_{i j}$, which can be written in the form

$$
\Psi_{i j}=\left\langle v_{i j}\right\rangle=\frac{\int \prod_{n} d \mathbf{u}_{n} v\left(\left|\mathbf{R}_{i}-\mathbf{R}_{j}+\mathbf{u}_{i}-\mathbf{u}_{j}\right|\right) \exp \left(-\beta \sum_{n} \frac{1}{2} \phi_{n}\left|u_{n}\right|^{2}\right)}{\int \prod_{n} d \mathbf{u}_{n} \exp \left[-\beta \sum_{n} \frac{1}{2} \phi_{n}\left|u_{n}\right|^{2}\right]},
$$

where $\mathbf{R}_{i}$ represents the coordinates of the equilibrium position for atom $i, \mathbf{u}_{i}$ denotes the displacement from that equilibrium position, $\beta=1 / k_{B} T$, the angular brackets represents thermal averaging, $\phi_{n}$ is the effective force constant at site $n$, and the index $n$ runs over all atomic sites in the particle. Substituting (1) and (2), the effective potential reduces to 8

$\Psi_{i j}=D_{i j}\left\{\exp \left[-2 \alpha\left(R_{i j}-r_{i j}\right)\right]-2 \exp \left[-\alpha\left(R_{i j}-r_{i j}\right)\right]\right\}$,

with

$$
\begin{aligned}
& D_{i j}=D \exp \left[-\frac{\alpha^{2}}{\beta}\left(\phi_{i}^{-1}+\phi_{j}^{-1}\right)\right], \\
& R_{i j}=\left|\mathbf{R}_{i}-\mathbf{R}_{j}\right|, \\
& r_{i j}=r_{0}+\frac{3 \alpha}{2 \beta}\left(\phi_{i}^{-1}+\phi_{j}^{-1}\right) .
\end{aligned}
$$

The two unknowns, $\phi_{i}$ (the effective force constant) and $\boldsymbol{R}_{i j}$ (the equilibrium interatomic distance), are determined self-consistently by the condition for minimum energy

$$
\sum_{j} \nabla_{R_{i}} \Psi_{i j}=0
$$

and the definition

$$
\phi_{i}=\frac{1}{3} \sum_{j} \nabla_{R_{i}}^{2} \Psi_{i j}
$$

Assuming a linear dependence of $R_{i j}$ on the temperature $T$, it can be shown that ${ }^{10}$

$$
\left.R_{i j} \approx R_{i j}\right|_{T=0}+\frac{3 \alpha}{2 \beta}\left(\phi_{i}^{-1}+\phi_{j}^{-1}\right) .
$$

For a given lattice structure, $\left.R_{i j}\right|_{T=0}$ can be found by solving Eq. (4a). Substitution of Eq. (5) into Eq. (3) then yields $\Psi_{i j}\left(\phi_{i}, \phi_{j}\right)$, enabling Eq. (4b) to be solved iteratively by Newton's method. This results in a self-consistent determination of $\left\{\phi_{i}\right\}$, which in turn defines the dynamical behavior of the atoms in the particle.

To study lattice softening in small Fe particles, we start with the bcc structure of $\mathrm{Fe}$ and use the method described above to obtain the effective force constants $\left\{\phi_{i}\right\}$ as a function of particle size. For simplicity, the shape of the particle is taken to be a cube whose volume equals the volume of a sphere of diameter $d$. We used parameters for the Morse potential that have previously been obtained for bulk Fe. ${ }^{11}$ The mean-square displacement of the atoms is then given by $\left\langle u_{i}^{2}\right\rangle=1 / \beta \phi_{i}$, which, when averaged over all the atoms in the particle, yields $\overline{\left\langle u^{2}\right\rangle}=\overline{\phi_{i}^{-1}} \mathrm{kT}$. The Einstein temperature $\Theta_{E}$ and the Debye temperature $\Theta_{D}$ are both related to the meansquare displacement by

$$
\overline{\left\langle u^{2}\right\rangle} \propto \frac{T}{\Theta_{E}^{2}} \propto \frac{T}{\Theta_{D}^{2}} .
$$

Thus, calculating $\left[{\overline{\left\langle u^{2}\right\rangle}}_{\infty} /{\overline{\left\langle u^{2}\right\rangle_{d}}}^{1 / 2}\right.$ using the Einstein model yields $\Theta_{E}(d) / \Theta_{E}(\infty)$, which is identical to $\Theta_{D}(d) / \Theta_{D}(\infty)$, the normalized Debye temperature as a function of particle size. The results of these calculations will be shown together with the experimental data.

\section{EXPERIMENTAL METHOD}

In a Mössbauer experiment, one measures the recoilless absorption of characteristic $\gamma$ rays by the nuclei of interest within the sample (in this case $\mathrm{Fe}$ ). At a given temperature, the mean-square displacement $\left\langle x^{2}\right\rangle$ of the Mössbauer atom in the $\gamma$-ray beam direction determines the fraction $f$ of atoms that participate in the recoilless absorption:

$$
f=\exp \left[-\frac{E_{\gamma}^{2}\left\langle x^{2}\right\rangle}{(\hbar c)^{2}}\right),
$$

where $E_{\gamma}$ is the $\gamma$-ray energy, $\hbar$ is Planck's constant divided by $2 \pi$, and $c$ is the speed of light. In the harmonic oscillator approximation, a Thirring expansion of $\left\langle x^{2}\right\rangle$ can be written, ${ }^{12}$ for high $T$, in terms of weighted mean frequencies (or frequency moments) $\omega(n)$ :

$$
\begin{aligned}
\left\langle x^{2}\right\rangle=\frac{k_{B} T}{M} & {\left[\frac{1}{\omega^{2}(-2)}+\frac{1}{12}\left(\frac{\hbar}{k_{B} T}\right]^{2}\right.} \\
& \left.-\frac{1}{720}\left[\frac{\hbar}{k_{B} T}\right]^{4} \omega^{2}(2)+\cdots\right],
\end{aligned}
$$

where $\omega(n)=\left[\sum_{i} b_{i}^{2} \omega_{i}^{n}\right]^{1 / n}, \omega_{i}$ are the normal mode frequencies, and $M$ is the mass of the Mössbauer atom. In the high-temperature limit

$$
\left\langle x^{2}\right\rangle \approx \frac{k_{B} T}{M} \frac{1}{\omega^{2}(-2)} .
$$

Therefore, measuring $f$ at high temperatures yields $\omega(-2)$, the $(-2)$ frequency moment of the phonon distribution. Assuming a Debye phonon distribution, this 
establishes a relation between $f$ and the Debye temperature $^{13}$

$$
\ln (f)=\frac{-3 E_{\gamma}^{2}}{M c^{2} k_{B} \Theta_{D}^{2}} T+C \quad\left(T>\Theta_{D} / 2\right),
$$

where $C$ is a constant. On a typical plot of Mössbauer data showing $\gamma$-ray absorption versus shift velocity, $f$ is proportional to the area $\boldsymbol{A}$ under the absorption peaks, thus $d(\ln f) / d T=d(\ln A) / d T$. For an ${ }^{57} \mathrm{Fe}$ experiment, this leads to

$$
\Theta_{D} \approx-11.6(d \ln A / d T)^{-1 / 2} \quad\left(T>\Theta_{D} / 2\right) .
$$

Hence $\Theta_{D}$ can be directly obtained from the slope of a $\ln A$-vs- $T$ curve, without the need to determine the absolute value of $f$. The high-temperature requirement $\left(T>\Theta_{D} / 2\right)$ amounts to $T>200 \mathrm{~K}$, since $\Theta_{D} \approx 400 \mathrm{~K}$ for Fe. We must here mention that Debye temperatures obtained from Mössbauer spectroscopy measurements (or any given experimental method) are often slightly different from those measured by other methods because the measured quantity in each case results from a different weight of the phonon distribution. ${ }^{6}$ In our case $\Theta_{D}$ may be thought of as a "characteristic Mössbauer Debye temperature," whose absolute value may be strictly compared only to values also measured by Mössbauer spectroscopy. If one does not want to assume a particular phonon distribution, then the experiment still measures changes in the fundamental quantities $\left\langle x^{2}\right\rangle$ and $\omega(-2)$.

The samples used in this study are $\mathrm{Fe}-\mathrm{SiO}_{2}$ and $\mathrm{Fe}$ $\mathrm{Al}_{2} \mathrm{O}_{3}$ granular materials, containing well-isolated $\mathrm{Fe}$ particles with average sizes below $3 \mathrm{~nm}$, smaller than any other Fe particles used for lattice dynamics studies to date. They were prepared by rf sputtering of composite $\mathrm{Fe}-\mathrm{SiO}_{2}$ and $\mathrm{Fe}-\mathrm{Al}_{2} \mathrm{O}_{3}$ targets onto Kapton and glass substrates at ambient temperature, with 4 mTorr of argon as the sputtering gas. Such materials have been used extensively in recent years to study magnetism in small particles, ${ }^{14}$ and present several distinct advantages for use in this study. First, the rigid amorphous $\mathrm{SiO}_{2}$ or $\mathrm{Al}_{2} \mathrm{O}_{3}$ matrix prevents any motion of the particles as a whole, which can completely obscure the Mössbauer effect (see discussion below). Second, they are structurally stable well above room temperature, a necessity for obtaining Mössbauer data in the high-temperature range discussed above [in contrast with other small particle fabrication techniques such as inert gas matrix isolation, where the solidified gas that forms the matrix must be kept at cryogenic temperatures; in such cases, structural determination by $\mathrm{x}$-ray or transmission electron microscopy (TEM) is also exceedingly difficult]. Third, and most importantly, each sample exhibits a narrow distribution of grain sizes. The average size is dependent on the metal volume fraction, the substrate temperature, the sputtering gas pressure, and the nature of the amorphous matrix. The matrix also protects the particles from uncontrolled oxidation once the samples are exposed to the atmosphere.

For our samples, TEM micrographs (Fig. 1) revealed average diameters of 1.5 and $2.5 \mathrm{~nm}$ for $\mathrm{Fe}-\mathrm{SiO}_{2}$ with 15 and $30 \mathrm{vol} \% \mathrm{Fe}$, respectively, and 1.4 and $1.7 \mathrm{~nm}$ for $\mathrm{Fe}-$

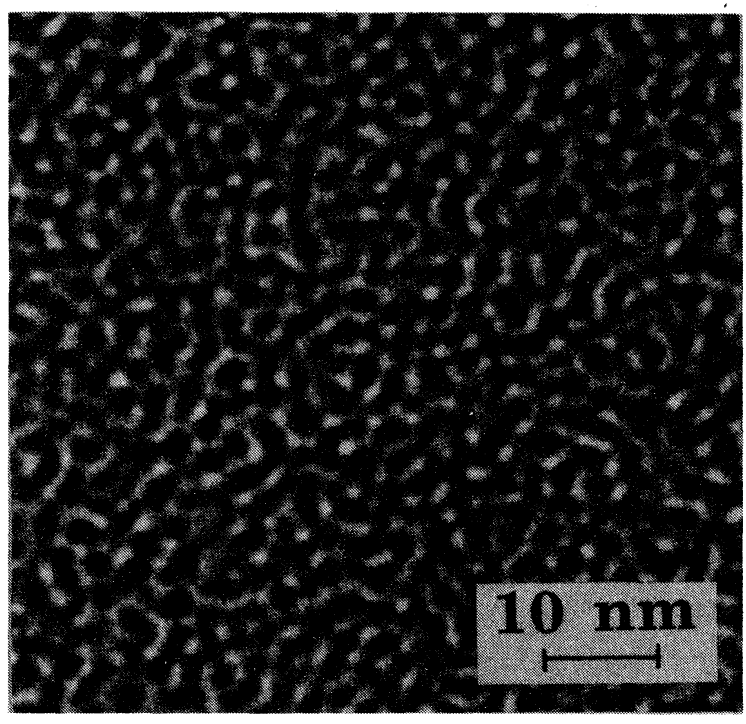

FIG. 1. Transmission electron micrograph of a $\mathrm{Fe}-\mathrm{SiO}_{2}$ granular material. The dark areas are crystalline $\mathrm{Fe}$ particles, while the light areas are amorphous $\mathrm{SiO}_{2}$. For this sample the Fe volume fraction is $30 \%$, with an average particle size of 2.5 nm.

$\mathrm{Al}_{2} \mathrm{O}_{3}$ with 18 and 35 vol \% $\mathrm{Fe}$, respectively. Although TEM micrographs are very useful for determining the average particle size, it is difficult to estimate from them the separation between the particles. Not all the particles on the picture are in sharp focus; consequently particle images from slightly different depths (with slightly different focus) will seem to overlap when looking through the samples. Instead, we also rely on the results of numerous studies of percolation-related phenomena (such as electrical conductivity and magnetic coercivity), which show that the percolation volume fraction $p_{c}$ in these granular systems is close to $60 \%$ (Ref. 15). The present samples, whose volume fractions are between 15 and $35 \%$, are thus well below this threshold and consist of physically isolated particles. This microstructure is fundamentally different than that of materials often referred to as "nanocrystalline materials." Such materials are usually single-element or alloy materials containing high densities of defects, whose distinct properties come from the fact that a majority of the atoms reside at grain boundaries. ${ }^{16}$ These nanocrystalline materials have also been the subject of Mössbauer spectroscopy studies. ${ }^{17}$ In our granular Fe samples, the Fe nanocrystals are physically separated by the amorphous insulating matrix of $\mathrm{SiO}_{2}$ or $\mathrm{Al}_{2} \mathrm{O}_{3}$.

The bcc $(\alpha-\mathrm{Fe})$ structure of the $\mathrm{Fe}$ particles was confirmed by $\theta-2 \theta$ x-ray-diffraction analysis, which revealed an unambiguous bcc pattern with considerable finite-size broadening (Fig. 2). Thus, despite the very small particle size, no structural transformation (to, for example the face-centered-cubic $\gamma$-Fe structure) is seen in our samples. Rather, the bulk crystalline structure is 


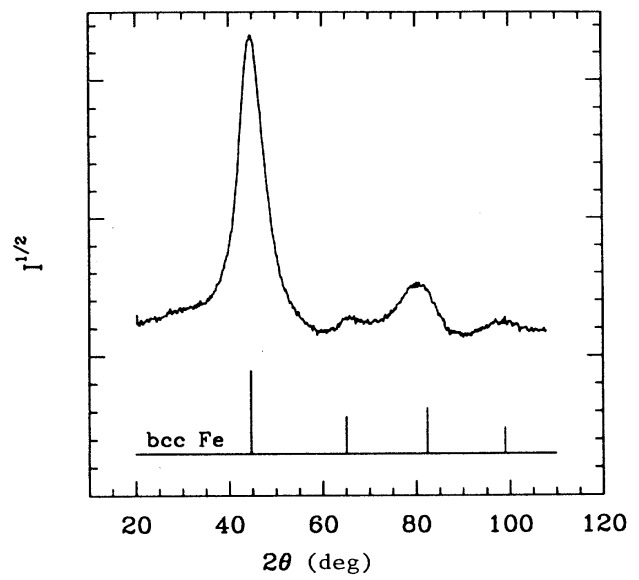

FIG. 2. X-ray-diffraction pattern of a $\mathrm{Fe}_{35}$ vol $\%-\mathrm{Al}_{2} \mathrm{O}_{3}$ sample. The pattern for bec $\alpha-\mathrm{Fe}$ is shown below for comparison.

retained. Of course, for atoms at the surface of particles, a cubic metallic structure cannot be strictly maintained. Thus we expect some interfacial Fe atoms to be in a highly perturbed electronic environment (without cubic symmetry), or to be oxidized. Fortunately, those atoms have distinct signatures in the Mössbauer spectra and can therefore be excluded from the analysis.

\section{RESULTS}

In Fig. 3 we show the Mössbauer spectrum of the $\mathrm{Fe}_{30 \text { vol } \%}-\mathrm{SiO}_{2}$ sample $(2.5-\mathrm{nm}$ particles) at $4.2 \mathrm{~K}$. A

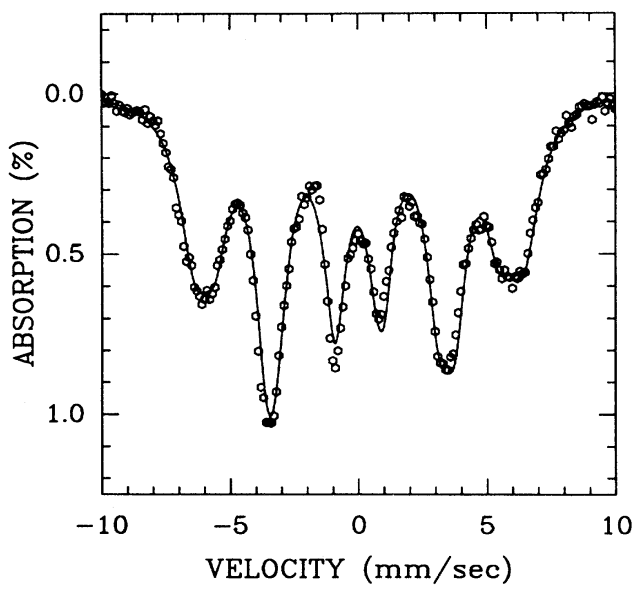

FIG. 3. Mössbauer spectrum of $\mathrm{Fe}_{30}$ vol $\%-\mathrm{SiO}_{2}$ (2.5-nm particles) at $T=4.2 \mathrm{~K}$. The fit (solid lines) assumes two contributions to the spectrum. broad, magnetically split six-line pattern is seen for this and all other samples, with no evidence of a central paramagnetic contribution. The width and slight asymmetry of the lines attests to multiple contributions to the spectrum. A good fit to this data (solid line in Fig. 3) is obtained if one assumes two main contributions. One is characterized by a magnetic hyperfine field $\left(H_{\mathrm{hf}}\right)$ close to the bulk Fe value (330-350 kOe). The other has a higher $H_{\mathrm{hf}}$ (380-400 kOe), which can be interpreted as oxide and/or surface contribution. One must note that these surface fields are still much smaller than the hyperfine fields of either $\mathrm{Fe}_{2} \mathrm{O}_{3}(\approx 530 \mathrm{kOe})$ or $\mathrm{Fe}_{3} \mathrm{O}_{4}(\approx 475 \mathrm{kOe}){ }^{18}$ Therefore extensive oxidation of the particle surface can certainly be ruled out. The higher $H_{\mathrm{hf}}$ may also originate from the reduced coordination number for surface $\mathrm{Fe}$ atoms, which results in higher magnetic moments. This phenomenon has already been observed in Fe thin films. ${ }^{19}$

The occurrence of superparamagnetic relaxation in small particle systems is well documented. ${ }^{20}$ The particles in our samples are small enough that they contain a single magnetic domain, and all display superparamagnetic behavior above a blocking temperature $T_{B} \approx 150 \mathrm{~K}$ (as measured by Mössbauer spectroscopy). Thus at room temperature and above, magnetic hyperfine interactions do not appear in the Mössbauer spectra of these samples. In the absence of hyperfine splitting, metallic $\mathrm{Fe}$ atoms (in a symmetric, cubic environment) yield a single unsplit absorption peak. In contrast, any oxidized $\mathrm{Fe}$, or highly perturbed Fe near the surface, is also subjected to an electric quadrupole interaction and will give rise to a split "doublet" peak. This fact enables us to distinguish between core and surface $\mathrm{Fe}$ atoms in our particles. As an

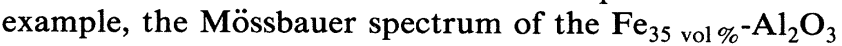
(1.7-nm particles) sample at $300 \mathrm{~K}$ is shown in Fig. 4. Similar spectra were obtained for all samples between 300 and $500 \mathrm{~K}$, a region where the high-temperature approximation is clearly valid. The asymmetrical spectrum, again, is indicative of multiple contributions. The line

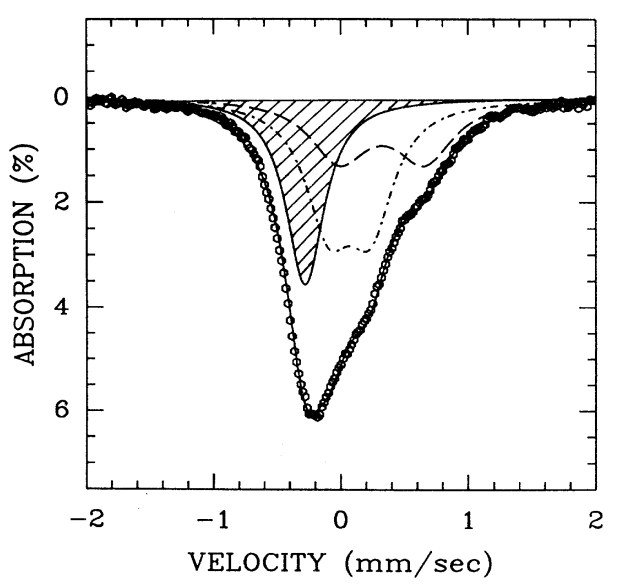

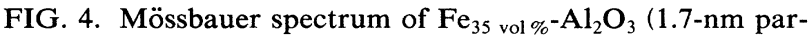
ticles) at $T=300 \mathrm{~K}$. Individual contributions to the fitted spectrum are shown above the data. 
shape on the negative velocity side is sharp and is due to the presence of a single line. The broad shoulder on the positive velocity side is poorly resolved, but indicates an overlap of several lines. To fit this data, we used a leastsquares fitting of Lorentzian line shapes, with all parameters allowed to vary freely, except that any doublet contributions were restricted to being symmetrical. The combination of line shapes used to fit a spectrum cannot, of course, simply be chosen to provide the closest fit to that particular spectrum. Rather, a successful fit must result in consistency of the fitted parameters for various samples and temperatures. It was determined that, in addition to the single line, two quadrupole doublet contributions were necessary and sufficient to obtaining a consistent fit for all samples at all temperatures.

Above the actual data (circles) in Fig. 4 we show the individual contributions to the spectrum. The line through the data is the sum of the three contributions, and illustrates the excellent fit obtained. The picture here is that each $\mathrm{Fe}$ grain consists of a core of metallic cubic $\mathrm{Fe}$ (single line) with an outer shell of atoms without cubic symmetry (two doublets). It is clear from the relative areas of each contribution that a large proportion of the spectrum results from surface atoms. This is consistent with the fact that for such small grain sizes a single surface layer can include more than $50 \%$ of the total number of atoms in the particle. The center shifts $(C$, which is the sum of the isomer shift and the second-order Doppler shift) of the three contributions and quadrupole splittings $(\Delta)$ for the two doublets are shown in Table I, where we see that consistent fits are obtained from sample to sample. There is a small negative center shift $(\approx-0.27 \mathrm{~mm} / \mathrm{sec}$ with respect to bulk $\mathrm{Fe})$ for the metallic $\mathrm{Fe}$ peak in all samples. Although this shift is significant, it is not unexpected, as the center shift is sensitive to local static and dynamical disturbances (such as changes in the electron density at the nucleus and changes in the lattice dynamics), both of which are known to occur in nanometer-size particles (see Ref. 3 for a more detailed discussion). $C_{3}$ and $\Delta_{3}$ are typical of what is expected for $\mathrm{Fe}^{3+}$; thus limited surface oxidation may be present in this form. ${ }^{21,22}$ The other contribution may tentatively be assigned to metallic surface $\mathrm{Fe}$ atoms (as opposed to $\mathrm{Fe}^{2+}$, since $C$ and $\Delta$ for $\mathrm{Fe}^{2+}$ are both typically higher than those of $\mathrm{Fe}^{3+}$ ). Indeed recent work on ultrathin $\mathrm{Fe}$ films ${ }^{23}$ has shown that surface $\mathrm{Fe}$ atoms exhibit center shift and quadrupole splitting values close to what we find for $C_{2}$ and $\Delta_{2}$. However, it is beyond the

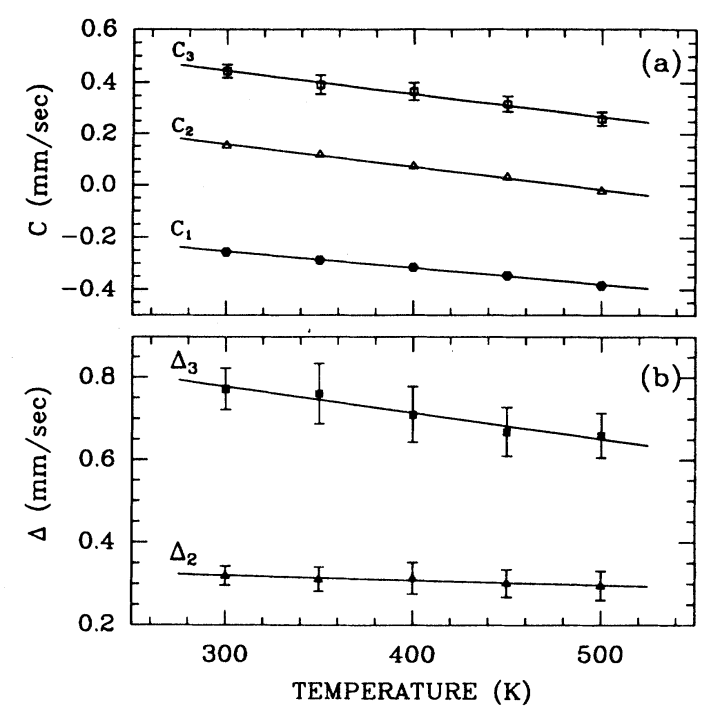

FIG. 5. (a) Center shifts $C$ of the three contributions to the Mössbauer spectrum of $\mathrm{Fe}_{15}$ vol\% $-\mathrm{SiO}_{2}$ as a function of temperature. Singlet (metallic Fe) site: filled circles. Doublet sites: open triangles and open squares. (b) Quadrupole splittings $\Delta$ of the two doublet sites for $\mathrm{Fe}_{15}$ vol \% $-\mathrm{SiO}_{2}$, as a function of temperature. The solid lines are least-squares fits to the data.

scope of the present paper to unambiguously assign the two doublets to specific surface and/or oxidation states.

The smooth variation of the fit parameters with temperature, an essential feature of any accurate fitting scheme, is shown in Fig. 5 for the $\mathrm{Fe}_{15}$ vol\% $-\mathrm{SiO}_{2}$ sample. It is particularly instructive to examine the temperature dependence of the center shift. At high temperatures $\left(T \gtrsim \Theta_{D}\right)$, the second-order Doppler shift (SOD) contribution to the center shift has the form

$$
\delta E / E=\left(-3 k_{B} T\right) /\left(2 M c^{2}\right),
$$

resulting in a linear temperature dependence with slope $-7.3 \times 10^{-4} \mathrm{~mm} / \mathrm{sec} \mathrm{K}$. For ${ }^{57} \mathrm{Fe}$, the isomer shift contributes little to the temperature dependence and thus the SOD contribution dominates. A linear least-squares fit of our $C$ data yields a slope, for the single peak, of

TABLE I. Mössbauer parameters (center shifts $C$ and quadrupole splittings $\Delta$ ) for the three sites fitted to the room-temperature spectra of $\mathrm{Fe}$ granular materials with different average particle sizes. The values of $C$ are with respect to $\alpha-\mathrm{Fe}$ at room temperature.

\begin{tabular}{ccccccc}
\hline \hline $\begin{array}{c}\text { Sample } \\
(\text { vol \%) }\end{array}$ & $\begin{array}{c}d_{\text {ave }} \\
(\mathrm{nm})\end{array}$ & $\begin{array}{c}C_{1} \\
(\mathrm{~mm} / \mathrm{sec})\end{array}$ & $\begin{array}{c}C_{2} \\
(\mathrm{~mm} / \mathrm{sec})\end{array}$ & $\begin{array}{c}\Delta_{2} \\
(\mathrm{~mm} / \mathrm{sec})\end{array}$ & $\begin{array}{c}C_{3} \\
(\mathrm{~mm} / \mathrm{sec})\end{array}$ & $\begin{array}{c}\Delta_{3} \\
(\mathrm{~mm} / \mathrm{sec})\end{array}$ \\
\hline $\mathrm{Fe}_{15}\left(\mathrm{SiO}_{2}\right)_{85}$ & 1.5 & -0.25 & +0.15 & 0.32 & +0.44 & 0.77 \\
$\mathrm{Fe}_{30}\left(\mathrm{SiO}_{2}\right)_{70}$ & 2.5 & -0.26 & +0.12 & 0.36 & +0.43 & 0.77 \\
$\mathrm{Fe}_{18}\left(\mathrm{Al}_{2} \mathrm{O}_{3}\right)_{82}$ & 1.4 & -0.28 & +0.12 & 0.38 & +0.38 & 0.66 \\
$\mathrm{Fe}_{35}\left(\mathrm{Al}_{2} \mathrm{O}_{3}\right)_{65}$ & 1.7 & -0.28 & +0.07 & 0.33 & +0.32 & 0.66 \\
\hline \hline
\end{tabular}




$$
(-6.34 \pm 0.17) \times 10^{-4} \mathrm{~mm} / \mathrm{sec} \mathrm{K},
$$

very close to the theoretical value above. In fact, it is nearly identical to the value of

$$
(-6.5 \pm 0.1) \times 10^{-4} \mathrm{~mm} / \mathrm{sec} \mathrm{K}
$$

we and others ${ }^{24}$ have obtained for the center shift of a six-line bulk $\mathrm{Fe}$ spectrum in the temperature range $300-500 \mathrm{~K}$. This temperature range is actually on the low side of the "high-temperature limit" (in this case $T \gtrsim \Theta_{D} \approx 400 \mathrm{~K}$ ), resulting in a value slightly lower than the theoretical asymptote. For the two doublet peaks, the fitted slopes are $(-8.74 \pm 0.27) \times 10^{-4}$ and $(-8.80 \pm 0.46) \times 10^{-4} \mathrm{~mm} / \mathrm{sec} \mathrm{K}$, also close to the theoretical SOD contribution. Similar results are obtained for all samples. This consistency of the fit parameters over the whole temperature range provides additional evidence that one singlet and two doublets give a very good account of the data.

Once the fitting of all three contributions is complete, we focus on the single line resulting from metallic cubic $\mathrm{Fe}$, which is the only contribution of interest, since only such atoms can be compared to bulk metallic Fe. This unique "finger printing" of the different $\mathrm{Fe}$ contributions in Mössbauer spectra allows the study of the lattice dynamics of metallic Fe only, by separately computing the area under the fitted singlet line at each temperature (shaded area in Fig. 4). Figure 6 shows a plot of the logarithm of the spectrum areas for metallic $\mathrm{Fe}$ (each normalized to the background count) versus temperature. For comparison, we also show the result of similar area measurements made on the six-line spectrum of a bulk $\alpha-\mathrm{Fe}$ film. As expected from Eq. (10), the data can be fitted to straight lines, and the slope obtained by a least-squares method is then used to compute the Debye temperature

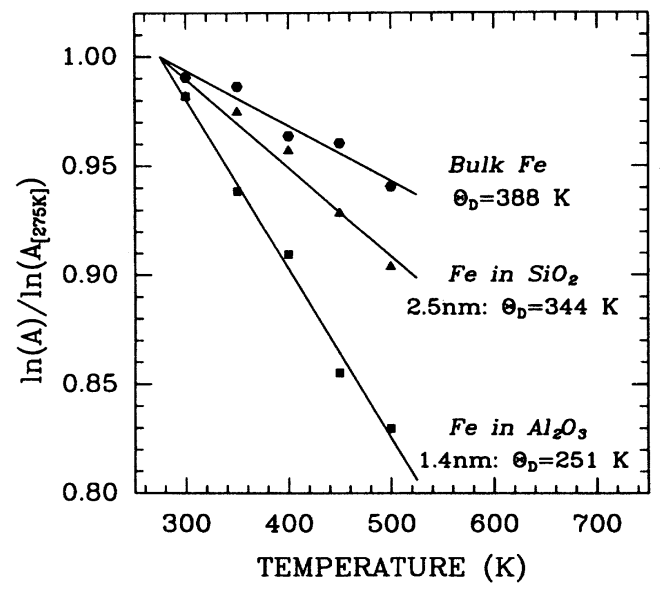

FIG. 6. Logarithm of Mössbauer absorption peak area, normalized to its value at $275 \mathrm{~K}$, vs temperature for bulk $\mathrm{Fe}$ and for metallic $\mathrm{Fe}$ in granular $\mathrm{Fe}-\mathrm{SiO}_{2}$ and $\mathrm{Fe}-\mathrm{Al}_{2} \mathrm{O}_{3}$ materials with different average particle sizes. The Debye temperatures $\Theta_{D}$ are computed from the slopes of the fitted lines. The uncertainty in $\ln A / \ln A(275 \mathrm{~K})$ at each data point is approximately \pm 0.01 .
$\Theta_{D}$, as explained previously. We find that $\Theta_{D}$ for bulk $\alpha-\mathrm{Fe}$ is $388 \pm 20 \mathrm{~K}$, in good accord with previously published results. ${ }^{25}$ However, for nanometer-size $\mathrm{Fe}, \Theta_{D}$ sharply decreases with decreasing particle size to $344 \pm 16$ and $259 \pm 18 \mathrm{~K}$ for 2.5 - and $1.5-\mathrm{nm}$ particles in $\mathrm{SiO}_{2}$, respectively, and to $264 \pm 16$ and $251 \pm 6 \mathrm{~K}$ for $1.7-$ and 1.4$\mathrm{nm}$ particles in $\mathrm{Al}_{2} \mathrm{O}_{3}$, respectively.

\section{DISCUSSION}

In Fig. 7, we plot $\Theta_{D}(d) / \Theta_{D}(\infty)$ vs particle size $d$ for theory and experiment. Both display the sharp decrease of $\Theta_{D}$ with particle size, in very good agreement. A decrease in excess of $30 \%$ for particle sizes near $1.5 \mathrm{~nm}$ has been observed. To our knowledge, this is the largest decrease in Debye temperature observed in nanometer-size particles by Mössbauer spectroscopy. It is also apparent that, in our samples, it is the Fe particle size (and not the matrix) that determines the observed reduction in $\Theta_{D}$. One could argue, for example, that if the particle is tightly bound to the surrounding matrix then the thermal properties of the matrix may strongly influence the thermal properties of the $\mathrm{Fe}$ grains. Both $\mathrm{SiO}_{2}$ and $\mathrm{Al}_{2} \mathrm{O}_{3}$ have complicated thermal properties, but the Debye temperature of the latter is significantly higher than that of $\mathrm{SiO}_{2}$, as indicated by specific heat measurements. ${ }^{26}$ In that case, the Debye temperature of the $\mathrm{Fe}_{35}$ vol \% $-\mathrm{Al}_{2} \mathrm{O}_{3}$ sample would be expected to be higher than that of the $\mathrm{Fe}_{30 \text { vol } \%}-\mathrm{SiO}_{2}$ sample. On the contrary, we find that the reverse is true, with the larger particle sizes in the latter sample resulting in a higher Debye temperature. Thus the reduction in $\Theta_{D}$ is intrinsic to the small particles, and not simply a reflection of the medium in which the particles are imbedded.

As the particle size is increased, $\Theta_{D}$ rises rapidly. A reasonable extrapolation of the experimental data would result in the bulk value being reached for particle sizes above $4 \mathrm{~nm}$, which agrees with the earlier null result on

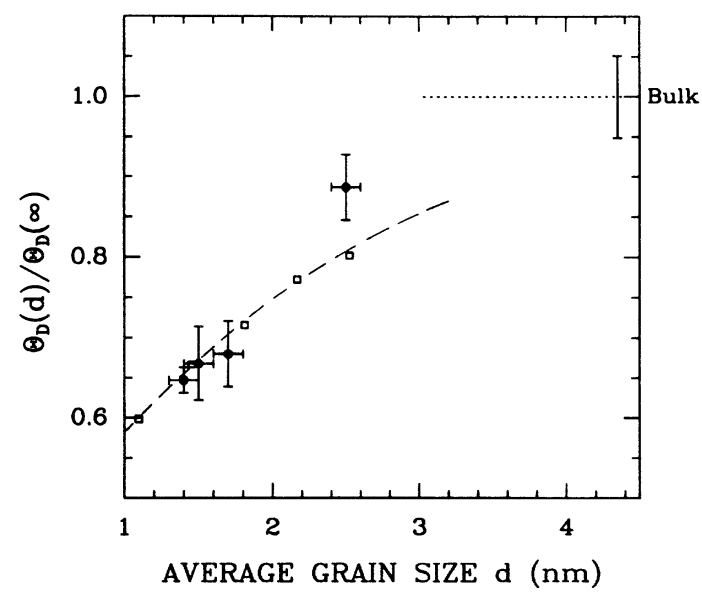

FIG. 7. $\Theta_{D}(d) / \Theta_{D}(\infty)$ vs particle size $d$ for our calculation (open squares) and Mössbauer measurements (filled circles). The uncertainty in the measurement on bulk $\alpha-\mathrm{Fe}$ is indicated on the dashed line. 


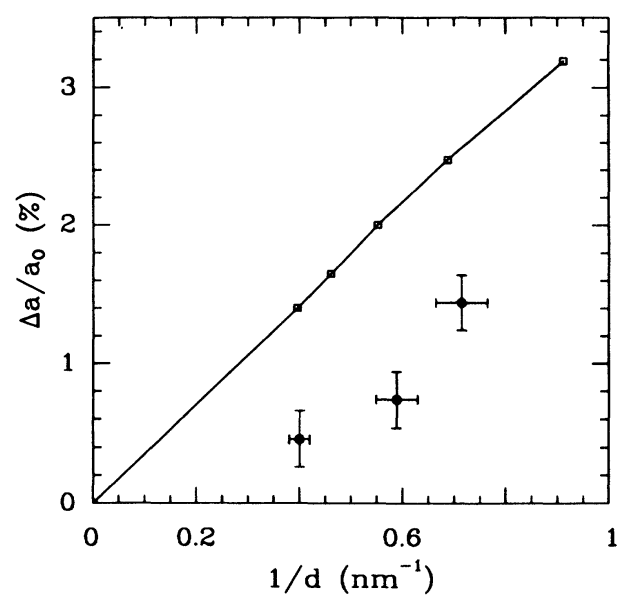

FIG. 8. Calculated (open squares) and measured (filled circles) change in the lattice constant $\Delta a / a_{0}$ of bcc Fe plotted as a function of inverse particle size $1 / d$. The solid line connects calculated points.

6.6-nm particles. ${ }^{10}$ Unfortunately, it is not possible in our case to fabricate samples covering this whole range of particle sizes, without either significantly widening the size distribution or obtaining a split spectrum due to the increased superparamagnetic blocking temperature. We also note that the sharp dependence of $\Theta_{D}$ on the particle size observed experimentally is somewhat less pronounced in our calculated results. As has been discussed in earlier studies, ${ }^{2}$ the rigid matrix (not taken into account in our theoretical treatment) is capable of significantly altering the lattice dynamics of ultrafine particles. It may, in this case, play a role in determining the exact behavior of $\Theta_{D}$ as a function of particle size.

Further evidence for the important role played by the matrix is found in results obtained on the crystalline lattice spacing. The lattice spacing $a_{0}$ as a function of particle size $d$ is calculated by determining the atomic separation, which minimizes the total energy [Eq. (4a)]. Results from the calculation are shown in Fig. 8, along with the lattice constants measured in our samples. The latter were obtained from $\theta-2 \theta$ x-ray diffraction, by measuring the position of the bcc [110] peak. Experimentally, we find a smaller expansion, on the order of $1.4 \%$ for the 1.4-nm particle, compared to the calculated result of about $2.5 \%$. Thus it appears that the presence of the matrix limits the relaxation process expected in freestanding small particles. The matrix, on the other hand, does play a positive and necessary role: "Freely dispersed" Fe particles, not bound in a rigid matrix, do show a large decrease in the apparent Debye temperature. ${ }^{27}$ This effect, however, is attributed to vibration or rotation of the particles as a whole, ${ }^{28}$ which obscures any reduction of $\Theta_{D}$ truly due to finite-size effects. Consequently, the use of Mössbauer spectroscopy to study phenomena intrinsic to the finite size must in most cases be restricted to bound particle systems. Therefore a calculation similar to ours, but including boundary conditions that simulate the effect of the matrix, although not yet available, would be of great interest.

\section{CONCLUSIONS}

In conclusion, careful Mössbauer-spectroscopy measurements on $\mathrm{Fe}-\mathrm{SiO}_{2}$ and $\mathrm{Fe}-\mathrm{Al}_{2} \mathrm{O}_{3}$ granular materials indicate a dramatic decrease in the Debye temperature of metallic $\mathrm{Fe}$ at the core of nanometer-size bcc particles. The magnitude of the decrease, in excess of $30 \%$ for particles around $1.5 \mathrm{~nm}$ in diameter, agrees very well with results from a self-consistent Einstein-model calculation using the Morse interaction potential. Results on the corresponding lattice spacings, however, suggest an important role for the binding matrix in limiting lattice relaxation in the particles.

\section{ACKNOWLEDGMENTS}

This work is supported by Office of Naval Research Contract Nos. N00014-91-J-1663 and N00014-88-K0003 . We also gratefully acknowledge A. Levy for numerous fruitful discussions during the early stages of the experimental research.
${ }^{1}$ See, e.g., W. P. Halperin, Rev. Mod. Phys. 58, 533 (1986).

${ }^{2}$ A. Tamura, K. Higeta, and T. Ichinokawa, J. Phys. C 15, 4975 (1982).

${ }^{3}$ R. L. Filler, P. Lindenfeld, T. Worthington, and G. Deutscher, Phys. Rev. B 21, 5031 (1980).

${ }^{4}$ K. Ohshima, A. Hayashi, and J. Harada, J. Phys. Soc. Jpn. 48, 1631 (1980).

${ }^{5}$ K. Ohshima, S. Yatsuya, and J. Harada, J. Phys. Soc. Jpn., 50, 3071 (1981)

${ }^{6}$ B. Kolk, in Dynamical Properties of Solids, edited by G. K. Horton and A. A. Maradudin (North-Holland, Amsterdam, 1984), Vol. 5.

${ }^{7}$ See, e.g., D. Schroeer, in Mössbauer Effect Methodology (Plenum, New York, 1970), Vol. 5, p. 141, and references therein.

${ }^{8}$ S. Akselrod, M. Pasternak, and S. Bukshpan, Phys. Rev. B 11, 1040 (1975).

${ }^{9}$ M. Havashi, I. Tamura, Y. Fukano, and S. Kanemaki, Surf.
Sci. 106, 453 (1981).

${ }^{10}$ M. Y. Zhou and Ping Sheng, Phys. Rev. B 43, 3460 (1991).

${ }^{11}$ T. Matsubara and K. Kamiya, Prog. Theor. Phys. 58, 767 (1977).

${ }^{12}$ R. M. Housley and F. Hess, Phys. Rev. 146, 517 (1966).

${ }^{13}$ R. H. Herber, Chemical Mössbauer Spectroscopy, edited by R. H. Herber (Plenum, New York, 1984), p. 199, and references therein.

${ }^{14}$ C. L. Chien, in Physical Phenomena in Granular Materials edited by G. D. Cody, T. H. Geballe, and Ping Sheng (Materials Research Society, Pittsburgh, 1990), Vol. 195, p. 411.

${ }^{15}$ B. Abeles, Appl. Solid State Sci. 6, 1 (1976).

${ }^{16}$ H. Gleiter, Prog. Mater. Sci. 33, 223 (1989).

${ }^{17}$ U. Herr, J. Jing, R. Birringer, U. Gonser, and H. Gleiter, Appl. Phys. Lett. 50, 472 (1987).

${ }^{18}$ See, e.g., J. Danon, in Chemical Applications of Mössbauer Spectroscopy, edited by V. I. Goldanskii and R. H. Herber 
(Academic, New York, 1968), p. 209.

${ }^{19}$ N. C. Koon, B. T. Jonker, F. A. Volkening, J. J. Krebs, and G. A. Prinz, Phys. Rev. Lett. 59, 2463 (1987).

${ }^{20}$ See, e.g., A. H. Morrish, The Physical Principles of Magnetism (Wiley, New York, 1965), p. 360.

${ }^{21}$ See, e.g., R. Ingals, F. Van der Woude, and G. A. Sawatzky, in Mössbauer Isomer Shifts, edited by G. K. Shenoy and F. E. Wagner (North-Holland, Amsterdam, 1978), p. 361.

${ }^{22}$ See, e.g., Mössbauer Spectroscopy, edited by D. P. E. Dickson and F. J. Berry (Cambridge University Press, Cambridge, England, 1986).

${ }^{23}$ C. J. Gutierrez, M. D. Wieczorek, H. Tang, Z. Q. Qiu, and J. C. Walker, J. Magn. Magn. Mater. (to be published).

${ }^{24}$ R. M. Housley and F. Hess, Phys. Rev. 164, 340 (1967).

${ }^{25}$ L. D. Lafleur and C. Goodman, Phys. Rev. B 4, 2915 (1971).

${ }^{26}$ Thermophysical Properties of Matter, edited by Y. S. Touloukian and E. H. Buyco (Plenum, New York, 1970), Vol. 5, pp. 24 and 202.

${ }^{27}$ G. von Eynatten and H. E. Bömmel, Appl. Phys. 14, 415 (1977).

${ }^{28}$ G. von Eynatten, T. Ritter, H. E. Bömmel, and K. Dransfeld, Z. Phys. B 65, 341 (1987). 


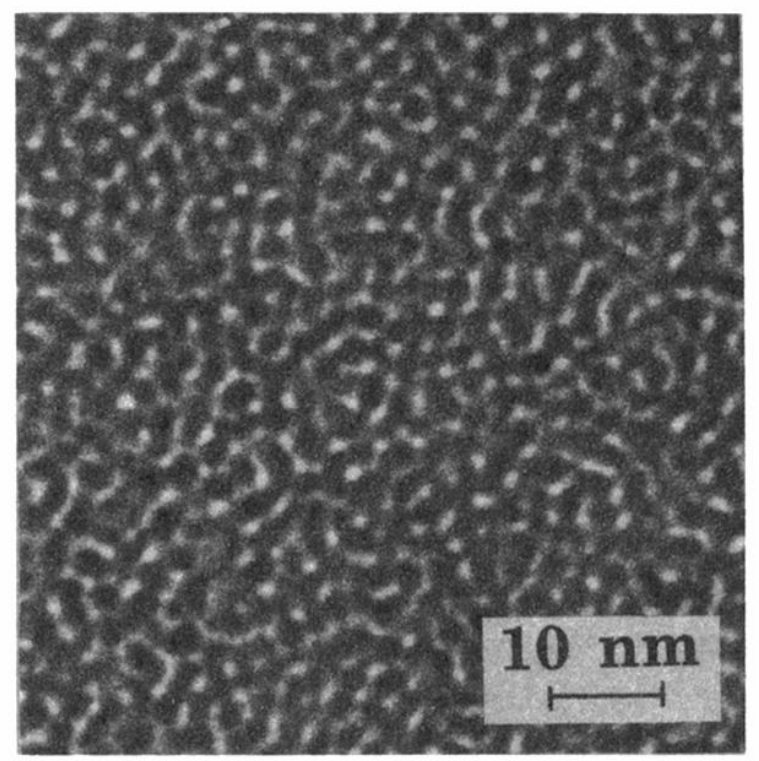

FIG. 1. Transmission electron micrograph of a $\mathrm{Fe}-\mathrm{SiO}_{2}$ granular material. The dark areas are crystalline $\mathrm{Fe}$ particles, while the light areas are amorphous $\mathrm{SiO}_{2}$. For this sample the $\mathrm{Fe}$ volume fraction is $30 \%$, with an average particle size of 2.5 nm. 